ARTIGO ORIGINAL

\title{
EPIDEMIOLOGIA DO VÍRUS DO DENGUE EM SÃO LUÍS, MARANHÃO, NO PERÍODO DE 2002 A 2012
}

\author{
Ana Cláudia Pinho de Carvalho ${ }^{1}$, Fernando Couto Portela ${ }^{2}$ Thiago Azevedo \\ Feitosa Ferro ${ }^{1,3}$ e Maria Rosa Quaresma Bomfim ${ }^{1}$
}

\section{RESUMO}

O presente estudo teve por objetivo relacionar a incidência do dengue com variáveis demográficas, temporais e meteorológicas, a fim de identificar as áreas com as mais elevadas incidências e determinar os sorotipos mais prevalentes, no período de 2002 a 2012, no Município de São Luís, MA. Os dados foram extraídos dos boletins dos casos de dengue até então notificados, disponíveis na Secretaria Municipal de Saúde e Vigilância Epidemiológica e Sanitária do Município de São Luís, MA. Foi calculada estatística descritiva para todas as variáveis relevantes, como idade, sexo, condições climáticas, distribuição dos sorotipos e índice de infestação predial (IIP) pela larva de Aedes aegypti. Um total de 21.986 casos de dengue foi notificado ao Sistema de Vigilância Epidemiológica da Secretaria de Saúde e Vigilância Epidemiológica de São Luís, MA, o que correspondeu a $34,3 \%$ dos casos notificados no estado do Maranhão durante o período estudado. A faixa etária mais atingida foi a de 20 a 49 anos, sem predomínio de sexo. A correlação entre os casos de dengue registrados e as condições meteorológicas, como pluviosidade, temperatura e umidade do ar, mostrou que a incidência de casos flutuou com essas variáveis climáticas. Assim, verificou-se ter ocorrido um aumento de casos de dengue durante o primeiro semestre dos anos estudados, que corresponde ao período chuvoso e de elevação de temperaturas, ao passo que, em intervalos de estiagem, a tendência foi de queda (meses de julho a dezembro). Nas mesmas condições, verificou-se um aumento da forma grave do dengue, como a febre hemorrágica do dengue, em população previamente exposta aos sorotipos 1,2 e 3. O IIP pelo A. aegypti foi maior no período de 2001 a 2007 e no ano de 2011. Nos anos em que foi registrada elevação dos índices de IIP, ocorreram os números mais elevados de casos de dengue no município de São Luis, MA.

DESCRITORES: Aedes aegypti; Dengue; incidência; vigilância epidemiológica.

\section{ABSTRACT}

Epidemiology of dengue virus from 2002-2012 in Sao Luis, Maranhao state, Brazil

\footnotetext{
1. Programa de Mestrado em Biologia Parasitária da Universidade Ceuma (UNICEUMA), São Luís, Maranhão, Brasil

2. Curso de Graduação em Medicina da UNICEUMA, São Luís, Maranhão, Brasil.

3. Universidade Federal do Maranhão, Programa de Pós-graduação Renorbio, São Luís, Maranhão, Brasil.

Endereço para correspondência: Maria Rosa Quaresma Bomfim, E-mail: mrqbomfim@yahoo.com.br
}

Recebido para publicação em: 14/6/2015. Revisto em: 14/3/2016. Aceito em: 2/7/2016. 
The purpose of this study is to relate the incidence of dengue with demographic, temporal and meteorological data, to identify the areas with the highest incidence of the disease and determine the most prevalent serotypes from 2002 to 2012, in São Luís, MA. Data were obtained from the reports of dengue cases so far reported, and available at the Municipal Health, Epidemiology and Sanitation Surveillance Office of São Luis, MA. Descriptive statistics were calculated for all the relevant variables, such as age, gender, weather conditions, distribution of dengue serotypes and building infestation index (BII) by $A$. aegypti larvae. A total of 21,986 dengue cases were notified to the surveillance system of São Luis-MA, corresponding to $34.3 \%$ of all cases reported in the state during the study period. The most affected age group was 20 to 49 years of age, with no gender predominance. The correlation between the reported dengue cases and weather conditions such as rainfall, temperature and humidity, showed that the incidence of dengue cases fluctuated with these meteorological variables. The number of cases increased during the first half of the study period, which corresponds to the rainy season and rises in temperature, while in the dry intervals, the trend was downward (from July to December). Under the same conditions, there was an increase in the severe form of dengue, namely dengue hemorrhagic fever (DHF) in the population previously exposed to serotypes 1, 2, and 3. The BII by A. aegypti was higher from 2001 to 2007 and in 2011. The highest numbers of dengue cases occurred concomitantly with the highest rates of BII recorded in Sao Luis, MA.

KEY WORDS: Aedes aegypti; dengue fever; incidence; epidemiological surveillance.

\section{INTRODUÇÃO}

O dengue vírus (DENV), agente etiológico da febre do dengue (FD) e da febre hemorrágica do dengue (FHD), é considerado o mais importante arbovírus que acomete o homem em todo o mundo (Murray et al., 2013). O DENV é transmitido pela picada de vetores artrópodes, mosquitos pertencentes ao gênero Aedes, sendo a espécie Aedes aegypti, o principal vetor (Kyle \& Harris, 2008; Luz et al., 2011).

Nos últimos vinte anos, $A$. aegypti disseminou o vírus do dengue em todo o Brasil, o que levou à ocorrência de várias epidemias em todas as suas regiões, incluindo as mais populosas. A incidência de dengue teve um crescimento significativo na década de 1990, atingindo um nível mais elevado em 1998, quando foram oficialmente registrados cerca de 530 mil casos (Barreto \& Teixeira, 2008; Ministério da Saúde, 2015). Houve uma redução acentuada em 1999, com o registro de 210 mil casos, e um pequeno crescimento em 2000, quando foram notificados 240 mil casos. Em 2002, foi registrado um aumento na incidência do dengue, alcançando 794.219 casos notificados. Nesse período, o número de mortes causadas pela febre hemorrágica do dengue (FHD) excedeu o número de mortes por malária pela primeira vez no País (Barreto \& Teixeira, 2008; Ministério da Saúde, 2015).

Conforme os boletins epidemiológicos disponíveis no portal do Ministério da Saúde, das cinco regiões brasileiras, a Região Nordeste é uma das mais afetadas pelo DENV e a população de todos os seus nove estados apresenta um risco muito elevado de infecção. Dos estados que a compõem, o Maranhão é um dos que têm apresentado maior número de casos de dengue (Ministério da Saúde, 2015). 
O sorotipo 1 (DENV-1) foi isolado pela primeira vez em São Luís, capital do estado do Maranhão, no ano de 1996; o sorotipo 2 (DENV-2), em 2001 (Neto \& Rebêlo, 2004). No ano de 2002, foram detectados os primeiros casos de dengue hemorrágico, o que coincidiu com a introdução e posterior prevalência do sorotipo 3 (DENV-3) (Neto \& Rebêlo, 2004). Desde então, os três sorotipos - DENV-1, DENV-2 e DENV-3 - cocircularam no município de São Luis-MA (Silva et al., 2010). A partir de maio de 2010, a incidência do dengue aumentou em 709\% em São Luís, em comparação com o mesmo período do ano anterior (O Estado do Maranhão, 2011).

O sistema de vigilância epidemiológica do dengue, no ano de 2011, identificou, até a $52^{\mathrm{a}}$ semana epidemiológica, três sorotipos circulantes no estado do Maranhão - DENV-1, DENV-2 e o DENV-4 -, não tendo sido registrada a circulação de DENV-3. Nesse mesmo período, sete amostras do sorotipo 4 foram isoladas pelo Instituto Evandro Chagas (IEC), a partir do soro de pacientes atendidos na rede básica de saúde das regiões: Tocantina (Estreito), região metropolitana (São Luís) e região do Munim (Rosário) (Governo do Maranhão, 2011).

Atualmente, a grande preocupação é que a recente introdução e circulação do DENV-4 abram portas para novas epidemias de dengue em São Luís, visto que a grande maioria da população ainda não teve contato com este sorotipo (Dantas \& Freitas, 2011). Vale ressaltar que a cocirculação de diferentes sorotipos tem aumentado o surgimento das formas clínicas mais graves da doença, a FHD ou síndrome do choque hemorrágico do dengue (SCHD) (Kyle \& Harris, 2008).

Portanto, são vários os fatores que limitam os estudos epidemiológicos para mensurar a incidência e a prevalência do dengue nos sete distritos sanitários do município de São Luís: a) as limitações dos programas de controle e de prevenção às epidemias de dengue; $b$ ) a ausência de uma vacina efetiva ao alcance de toda a população; c) as dificuldades na erradicação do mosquito A. aegypti; d) a introdução do sorotipo 4 em outros municípios maranhenses; e) a escassez de estudos sobre a incidência e prevalência do dengue no estado do Maranhão.

Diante dessas considerações, este estudo objetivou caracterizar o perfil epidemiológico das infecções por dengue no município de São Luís, MA, tendo por base a distribuição dos casos notificados no período de 2002 a 2012, relacionando a incidência do dengue com variáveis demográficas, temporais e meteorológicas para identificar as áreas com incidência mais elevada e os sorotipos mais prevalentes.

\section{MATERIAL E MÉTODOS}

Trata-se de um estudo observacional descritivo e retrospectivo, realizado no município de São Luís, MA, Brasil, no período de 2002 a 2012. 
O município ocupa uma área de $834.785 \mathrm{~km}^{2}$ do estado do Maranhão, situa-se $2^{\circ}$ ao sul do Equador, nas coordenadas geográficas latitude S $2^{\circ} 3^{\prime}$, longitude W $44^{\circ} 16$ e altitude 24 metros. A cidade de São Luís tem clima do tipo tropical, quente e semiúmido da zona equatorial com duas estações distintas: o verão, de julho a dezembro, e o inverno, de janeiro a junho. $O$ verão é quente e seco com ventos frescos e o inverno é chuvoso. Possui média pluviométrica de 1953 $\mathrm{mm}^{3}$. A cidade de São Luís, no período estudado, tinha população estimada de 1.064.197 habitantes, segundo os censos de 2000 (Instituto Brasileiro de Geografia e Estatística, 2000), de 2010 (Instituto Brasileiro de Geografia e Estatística, 2010) e Nota Técnica 01/2014 (Instituto Brasileiro de Geografia e Estatística, 2014).

Por motivos operacionais e administrativos, o município de São Luís foi dividido pela Secretaria Municipal de Saúde de São Luís (SEMUS) em sete Distritos Sanitários de Saúde (DS): Vila Esperança, Bequimão, Itaqui Bacanga, Centro, Cohab, Coroadinho e Tirirical (Silva \& Rangel, 2014). Os DS Coroadinho e Vila Esperança têm maior número de localidades rurais (Neto et al., 2006).

Os dados utilizados no presente estudo foram coletados nos boletins dos casos de dengue notificados entre 2002 e 2012, armazenados no Sistema de Agravos Notificáveis (SINAN) da Secretaria Municipal de Saúde e Vigilância Epidemiológica e Sanitária do Município de São Luís, MA. Já os dados meteorológicos foram obtidos das planilhas de dados diários do Instituto Nacional de Pesquisas Espaciais (INPE)/PCD meteorológica da cidade de São Luís - MA (2 $35^{\circ} \mathrm{S} ; 44^{\circ} 12^{\prime} \mathrm{W}$ com $\left.62 \mathrm{~m}\right)$.

Para a análise estatística dos dados, foi utilizado o programa BioEstat 5.0 (Ayres et al., 2007). As análises consideraram as seguintes variáveis: (a) temporal ou relacionada ao total do número de casos de dengue ocorridos no período de tempo estudado e notificados à Secretaria Municipal de Saúde e Vigilância Epidemiológica e Sanitária do Município de São Luís, MA; (b) meteorológica para analisar uma possível relação entre pluviosidade, temperatura e número de casos de dengue por ano, utilizando-se os dados do (INPE)/PCD meteorológica; (c) demográfica ou relacionada ao número de casos de dengue por distrito sanitário que foram notificados à Secretaria Municipal de Saúde e Vigilância Epidemiológica e Sanitária do Município de São Luís,MA; (d) distribuição anual dos sorotipos do dengue que circularam no período estudado, segundo os dados constantes nos arquivos da Secretaria Municipal de Saúde e Vigilância Epidemiológica e Sanitária do Município de São Luís, MA; (e) índice de infestação predial, relacionado com a média do número de criadouros do mosquito transmissor do vírus dengue, segundo os dados dos arquivos da Secretaria Municipal de Saúde e Vigilância Epidemiológica e Sanitária do Município de São Luís, MA.

$\mathrm{O}$ índice de infestação predial (IIP) corresponde à proporção de imóveis com larvas de $A$. aegypti em relação ao total de imóveis inspecionados 
(Monteiro et al., 2009). A correlação de Pearson analisou a variação dos dados meteorológicos em relação à frequência de casos da doença durante os meses. Os resultados foram descritos por meio de médias, desvio padrão, medianas, proporções e distribuições de frequência. $O$ teste do qui-quadrado $\left(\chi^{2}\right)$ foi utilizado para analisar as diferenças na proporção de casos por faixa etária, estação do ano e sexo, sendo consideradas diferenças significativas quando $\mathrm{p}<0,05$.

Este estudo foi aprovado pelo Comitê de Ética em Pesquisa da Universidade Ceuma, Parecer Consubstanciado no 467.140/2013.

\section{RESULTADOS}

Um total de 21.986 casos de dengue foi notificado no município de São Luis, MA no período de 2002 a 2012, o que correspondeu a 34,3\% dos casos notificados em todo o estado nesse período. Nos dados obtidos da série histórica do dengue em São Luís, de 2002 a 2012, os anos de 2007 (aproximadamente 3.000 casos) e 2011 (mais de 5.000 casos) destacaram-se em relação aos demais $(\mathrm{F}=7.95, \mathrm{p}<0.0001)$. Na Figura 1A, é possível observar dois picos referentes a estes dois anos.

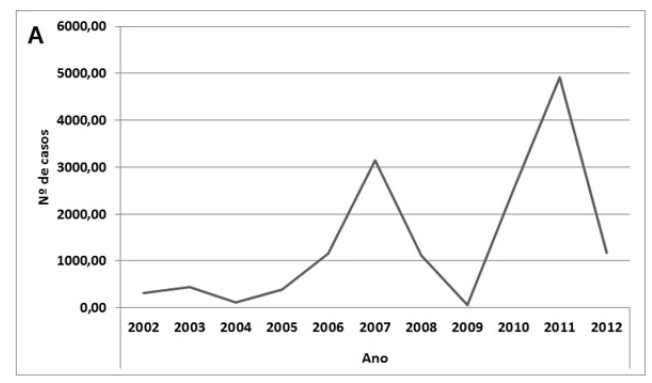

Figura 1A. Série histórica do número de casos de dengue notificados em São Luís, MA no período de 2002 a 2012.

Com relação à distribuição do número de casos de dengue, segundo o trimestre de cada ano, verificou-se um aumento crescente do número de casos notificados a partir do final do primeiro e segundo trimestre (valor de $\mathrm{p}<0,05$ ), que se manteve constante. Contudo, no início do terceiro trimestre se verificou maior incidência de casos de dengue nos períodos de julho a agosto $(\mathrm{F}=2,87, \mathrm{p}=0,0026)$. O número de notificações diminuiu no início do quarto trimestre $(\mathrm{H}=14,31, \mathrm{p}=0,0023)$. Ou seja, $16 \%$ dos casos ocorreram no primeiro trimestre, $41,3 \%$ no segundo, $35,5 \%$ no terceiro e $7 \%$ no quarto trimestre, conforme mostra a Figura 1B. 


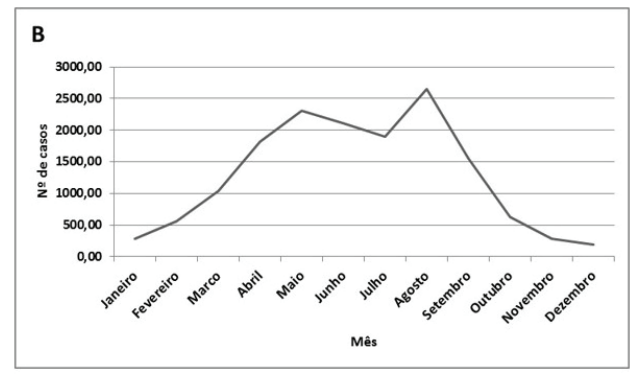

Figura 1B. Distribuição mensal dos casos de dengue notificados em São Luís, MA no período de 2002 a 2012.

A análise dos dados meteorológicos do número de casos notificados de dengue, no período de 2002 a 2012, em São Luís-Maranhão, com relação a temperatura, pluviosidade e umidade relativa do ar, mostrou que os números mais expressivos de casos de dengue corresponderam aos meses de maior pluviosidade em São Luís, verificando-se significância estatística $(p=0,003$ teste de Mann Whitney) conforme está demonstrado na Figura 2.

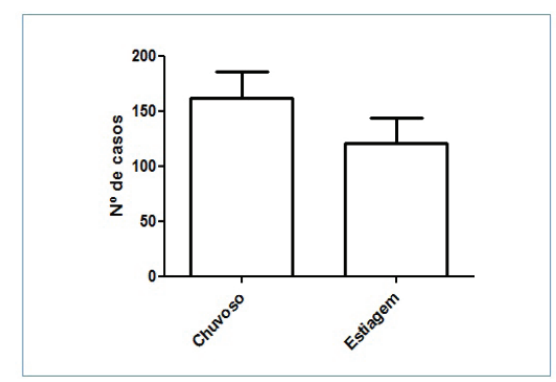

Figura 2. Correlação do número de casos com o período chuvoso (de janeiro a junho) e a estiagem (julho a dezembro) no período estudado.

Na análise da prevalência de casos de dengue na cidade de São Luís, MA, por distrito sanitário, região urbana e rural entre os anos de 2002 e 2012 (Kruskal-Wallis $=29,47, \mathrm{p}=0.0034$ ), notou-se que não houve diferença estatística na prevalência, exceto na região rural do terceiro distrito sanitário, denominado Coroadinho (Figura 3).

Com relação ao índice de infestação predial que foi notificado à Secretaria Municipal de Saúde e Vigilância Epidemiológica e Sanitária do Município de São Luís, MA, verificou-se que os criadouros das larvas do 
mosquito A. aegypti estavam distribuídos de forma desigual durante o período estudado, ou seja, houve um período entre 2008 e 2010 em que o número de registro de focos do mosquito foi menor. Nos anos de 2002, 2003, 2004, 2006, 2007 e 2011, foram registrados os índices mais elevados de infestação de residências do município de São Luís (Figura 4).

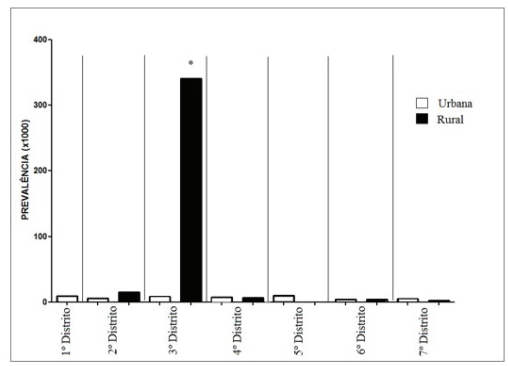

Figura 3. Distribuição do número de casos de dengue na cidade de São Luís, MA por distrito sanitário, região urbana e rural entre os anos de 2002 e 2012. *Valor de $\mathrm{p}=0,0034$.

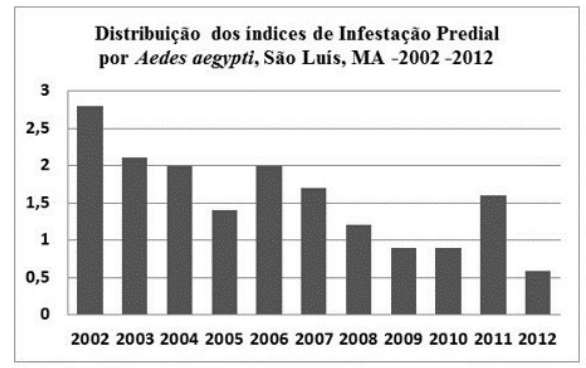

Figura 4. Distribuição dos índices de infestação predial por Aedes aegypti em São Luís-Maranhão no período de 2002 a 2012.

$\mathrm{Na}$ tabela observa-se os dados relacionados à evolução clínica do dengue nas pessoas que contraíram a infecção, evidenciando que a forma clássica foi mais presente e a que mais evoluiu para a cura. Os óbitos ocorridos nos anos de 2007 e 2011 coincidiram com registros de complicações relacionadas ao dengue e casos de FHD, justamente nos anos de pico máximo de casos de dengue. Com relação à distribuição de casos de dengue segundo a faixa etária e o sexo, no período analisado, observou-se que a faixa etária de 20 a 49 anos foi a mais atingida, com 7.360 casos no sexo masculino e 8.004 casos no sexo feminino, não havendo diferença significativa no número total de casos entre ambos os sexos. 


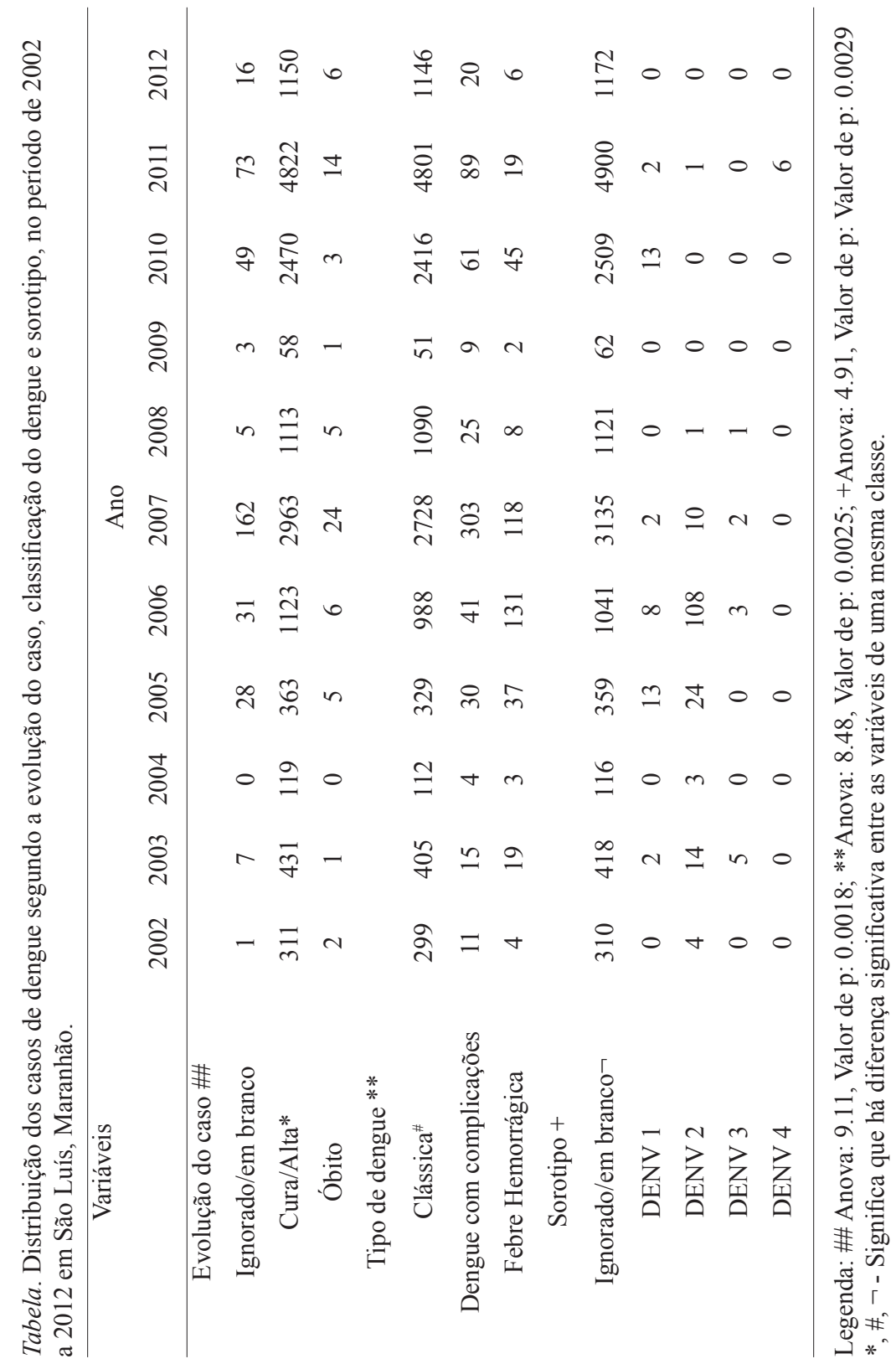




\section{DISCUSSÃO}

O dengue é uma doença infecciosa de etiologia viral e evolução aguda, tendo como principal vetor o artrópode Aedes aegypti. É considerada, atualmente, como a causa mais importante de doença viral transmitida por mosquito em regiões tropicais (Kyle \& Harris, 2008; Murray, 2013).

No presente estudo, o levantamento epidemiológico da série histórica do número de casos de dengue de 2002 a 2012, em São Luís, MA, revelou um número elevado em relação aos demais municípios que integram o estado. Em 2007, a epidemia se estabeleceu possivelmente pela introdução de um novo sorotipo em 2006, o DENV-3. Por outro lado, além da coexistência dos três sorotipos - DENV-1, DENV-2 e DENV-3 -, no ano de 2011 em São Luís houve a introdução do DENV-4. Tem-se observado que, após a introdução de um novo sorotipo do vírus do dengue em um determinado local, segue-se uma explosão epidêmica com elevadas taxas de incidência e grande número de casos (Barreto \& Teixeira, 2008). Se fosse possível fazer previsões razoáveis sobre as condições que afetam o desencadeamento desse processo, estratégias mais efetivas de prevenção poderiam ser elaboradas e implementadas. Entretanto, apesar das tentativas feitas para desenvolver modelos para previsão da ocorrência de epidemias de dengue, ainda não existem elementos que permitam um prognóstico seguro de curto prazo (Barreto \& Teixeira, 2008).

No período de 2008 a 2009, observou-se nos boletins de notificação uma diminuição nas taxas de incidência de dengue, possivelmente em decorrência de problemas operacionais como: falhas no sistema de informação, pouco interesse dos profissionais de saúde em efetuar a notificação e deficiências estruturais na assistência das unidades do Sistema Único de Saúde, o que faz com que a população, já ciente de como tratar o dengue clássico, não procure atendimento em tais unidades (Ministério da Saúde, 2015). Além disso, a incidência de casos de dengue flutua de acordo com as condições climáticas e está associada a fatores como aumento da temperatura, pluviosidade e umidade relativa do ar (Viana \& Ignotti, 2013).

O maior número de notificações de casos de dengue no período analisado foi registrado nos períodos chuvosos, ou seja, no primeiro semestre de cada ano. Os dados do presente estudo estão de acordo com relatos previamente registrados em São Luís, no período compreendido entre os anos de 1997 e 2002, quando foi observada correlação entre o índice pluviométrico e o aumento no número dos casos de dengue (Neto \& Rebêlo, 2004). Os autores responsáveis por tal estudo observaram maior frequência de casos na estação chuvosa $(83,8 \%)$, em detrimento do período de estiagem $(16,2 \%)$. Relataram também uma correlação positiva, ao longo dos anos, com precipitação pluviométrica $(\mathrm{r}=0,08415)$, umidade relativa $(\mathrm{r}=0,7606)$ e negativa com a temperatura $(r=-0,7838)$. 
É importante ressaltar que vários estudos têm demonstrado que a chuva exerce grande influência na determinação do período de ocorrência de surtos do dengue (Tauil, 2001; Glasser \& Gomes, 2002; Ribeiro et al., 2006; Fernandes et al., 2013; Viana \& Ignotti, 2013). Os chamados fatores abióticos favorecem tanto o aparecimento de depósitos naturais de água, que funcionam como criadouros naturais, como propiciam condições ideais de desenvolvimento das formas adultas do mosquito vetor (Ribeiro et al., 2006; Viana \& Ignotti, 2013).

Quanto à prevalência de casos de dengue na cidade de São Luís-MA, por distrito sanitário, região urbana e rural, entre os anos de 2002 e 2012, notouse que não houve diferença estatística na prevalência, exceto na região rural do terceiro distrito sanitário, denominado Coroadinho. Este distrito também já havia se destacado, em estudo anterior, como o segundo mais afetado (Neto \& Rebêlo, 2004). Outro aspecto relevante observado no presente estudo foi a homogeneidade do número de casos entre as classes sociais. Dado este que se contrapõe aos resultados de um estudo soroepidemiológico realizado em São Luís, no qual se verificou um número maior de casos nas classes com maior renda (Vasconcelos et al., 1999). Provavelmente, em contraste com anos anteriores, tenham aumentado o esclarecimento de toda população e a aceitação das visitas dos agentes de saúde.

Com relação ao índice de infestação predial do mosquito $A$. aegypti, foi observada uma distribuição desigual desse vetor durante o período estudado. Nos anos de 2002, 2003, 2004, 2006, 2007 e 2011, o serviço de vigilância entomológica registrou os mais elevados índices de infestação de residências em São Luís, MA. Tais resultados são coincidentes com os observados quanto à incidência de casos de dengue na capital durante este mesmo período. Além disso, concordam com os resultados de um estudo realizado em Teresina, PI, outra capital da Região Nordeste, onde os autores observaram que, nos anos de 2004 a 2006, foram elevados os índices de IIP em residências (Monteiro et al., 2009). Deve-se ressaltar que as precárias condições de infraestrutura sanitária e a explosão demográfica em áreas tropicais despontam como fatores importantes para a proliferação do vetor e, consequentemente, para a transmissão da doença. Inserem-se, neste contexto, os criadouros disponibilizados pelo homem como consequência da precariedade dos serviços de coleta de lixo e abastecimento de água (Tauil, 2001; Neto \& Rebêlo, 2004).

O dengue tem características clínicas variáveis desde as formas assintomáticas, subclínicas até casos fatais. Didaticamente elas têm sido divididas em quatro grupos, a saber: infecções assintomáticas; febre do dengue que, por sua vez, pode ser subdividida em dengue clássica; febre hemorrágica do dengue e síndrome do choque dengue e formas atípicas que simulam outras doenças febris, como hepatite e manifestações do sistema nervoso (Fonseca \& Figueiredo, 2009; Luz, 2011).

Neste estudo, verificou-se que a maioria das infecções por dengue evoluiu clinicamente para a cura, uma vez que a forma clássica foi a mais 
frequente. Isso está de acordo com a literatura - a FD é a forma mais comum com evolução benigna e autolimitada (Kyle \& Harris, 2008). No entanto, destaca-se que os óbitos ocorridos nos anos de 2007 e 2011 coincidiram com registros de complicações relacionadas ao dengue clássico e casos de FHD. Observou-se também que as complicações e os óbitos aconteceram nos anos de pico máximo de casos de dengue, o que pode ser explicado pela imunização prévia da população pelos sorotipos anteriores, favorecendo aparecimento das formas graves.

A causa do surgimento das formas graves do dengue tem sido objeto de pesquisas; sabe-se, no entanto, que estaria relacionada não apenas com a virulência da linhagem viral, mas também com a imunidade prévia aos outros sorotipos (Luz, 2011). A teoria da infecção sequencial pelos vírus do dengue caracteriza-se pela imunoamplificação dependente de anticorpos preexistentes e tem sido amplamente aceita (Mairuhu et al., 2004; Kyle \& Harris, 2008). Na teoria sequencial, a febre hemorrágica do dengue ocorre após epidemias de febre do dengue. É interessante observar que, no estudo realizado em São Luís, na década de 1990 os autores chamaram à atenção para o risco de ocorrência de febre hemorrágica se outros sorotipos fossem introduzidos em São Luís (Vasconcelos et al., 1999). Fato este observado em 2011, com a identificação do sorotipo 4 em São Luís e em outros municípios do estado do Maranhão (Secretaria Estadual de Saúde do Maranhão, 2014).

Com relação à distribuição de casos de dengue, segundo a faixa etária e o sexo, observou-se que a faixa etária de 20 a 49 anos foi a mais atingida. Resultado este semelhante aos encontrados por outros autores (Vasconcelos et al., 1999; Figueiredo et al., 2004). Convém ressaltar que essa faixa etária foi a mesma observada em outro estudo realizado no Maranhão (Neto \& Rebêlo, 2004). Quanto ao gênero, não ocorreu diferença significativa no número total de casos entre ambos os sexos, corroborando estudos prévios (Vasconcelos et al., 1999; Glasser et al., 2002; Neto \& Rebêlo, 2004).

Em conclusão, no presente estudo, verificou-se que as infecções pelo vírus do dengue vêm se mantendo constantes nas últimas décadas no município de São Luís, MA, alternando-se em anos epidêmicos e anos não epidêmicos. Embora a pesquisa tenha se limitado ao município de São Luís, os dados do Ministério da Saúde sobre o estado do Maranhão informam que os surtos apresentam características regionais quantitativas importantes, como o maior número de casos notificados na sua capital. Provavelmente, estas notificações estejam relacionadas com a maior facilidade de acesso da população aos serviços de saúde. A febre do dengue foi a forma mais notificada no período estudado, mas casos de FHD foram também registrados. Alguns fatores contribuíram para a ocorrência da FHD, tais como: a circulação simultânea de mais de um sorotipo, infecções recorrentes e introdução de sorotipo ainda não circulante. A introdução do sorotipo 4 tem provocado uma preocupação constante, visto que a grande maioria da população maranhense ainda está susceptível às infecções por 
este sorotipo e a reinfecção constitui um evento que potencializa o agravamento clínico do dengue. Em suma, São Luís, como muitas outras cidades brasileiras, enfrenta as limitações dos programas de controle e prevenção a epidemias de dengue, dificuldades diagnósticas e de erradicação do mosquito vetor.

\section{AGRADECIMENTOS}

Os autores agradecem à Dra. Maria do Socorro da Silva, da Secretaria Municipal de Saúde de São Luís, Maranhão, e a Afonso Luz, ambos da Vigilância Epidemiológica Municipal de São Luís-MA, pelo fornecimento de dados; à Fundação de Amparo à Pesquisa e Desenvolvimento Científico do Maranhão - FAPEMA projeto universal 01/2013 e à Universidade Ceuma, pelo apoio financeiro.

\section{REFERÊNCIAS}

1. Ayres MM, Ayres Jr, Ayres DL, Santos ASA. Aplicações estatísticas nas áreas de ciências biomédicas. 5ed. Belém, Pará: 2007. 359p.

2. Barreto ML, Teixeira MG. Dengue no Brasil: situação epidemiológica e contribuições para uma agenda de pesquisa. Estudos Avançados 22: 53-72, 2008.

3. Dantas LCM, Freitas IAS. 2011. Detecção do sorotipo viral quatro do dengue no estado do Maranhão. Disponível em: http://www.sbpcnet.org.br/livro/64ra/resumos/resumos/7076.htm. Acesso em: 12/03/2016.

4. Fernandes DR, Santos EA, Araújo AFDV, Zannoni C, Sardinha AHL, Rodrigues ZMR. Epidemiologia do dengue em São Luís-Maranhão, Brasil, 2000 a 2007. Cad Pesqui 2: 68-75, 2013.

5. Figueiredo RMP, Thatcher BD, Lima ML, Almeida TC, Alecrim WD, Guerra MVF. Doenças exantemáticas e primeira epidemia ocorrida em Manaus, Amazonas, no período de 1998-1999. Rev Soc Bras Med Trop 6: 476-479, 2004.

6. Fonseca BAL, Figueiredo LTM. Dengue. In: Roberto Focaccia, Ricardo Veronesi. Tratado de Infectologia. 4ed. São Paulo, Atheneu, 2009. p. 397-410.

7. Glasser CM, Gomes AC. Clima e sobreposição da distribuição de Aedes aegypti e Aedes albopictus na infestação do Estado de São Paulo. Rev Saúde Pública 36: 166-172, 2002.

8. Governo do Maranhão. 2011. Capacitação nas Ações de Vigilância Epidemiológica e Atenção ao Doente de Dengue. Disponível em: http://www2.ma.gov.br/index.php/tag/saude/page/18/. Acesso em: 12/03/2016.

9. Instituto Brasileiro de Geografia e Estatística. IBGE Censo Demográfico 2000. Características da População e dos Domicílios: Resultados do universo. Disponível em: http://www.ibge.gov. br/home/estatistica/populacao/censo2000/universo.php?tipo=31o/tabela13_1.shtm\&paginaat ual $=1 \& u f=21 \&$ letra $=$ S. Acesso em 12/03/2016.

10. Instituto Brasileiro de Geografia e Estatística. IBGE Censo Demográfico 2010. Disponível em: http://www.cidades.ibge.gov.br/xtras/perfil.php?lang=Acesso em 15/08/2015.

11. Instituto Brasileiro de Geografia e Estatística. IBGE. Gerência de Estudos e Análises da Dinâmica Demográfica - GEADD- Nota Técnica Estimativas da População dos Municípios Brasileiros com data de referência em $1^{\circ}$ de julho de 2014. Disponível em: http://www.ibge. gov.br/home/presidencia/noticias/pdf/analise_estimativas_2014.pdf. Acesso em 12/03/2016. 
12. Kyle JL, Harris E. Global Spread and Persistence of Dengue. Annu Rev Microbiol 62: 71-92, 2008.

13. Luz KG, Maciel JFR, Souza PM. Dengue clássico e febre hemorrágica do dengue: abordagem para o médico intensivista. Programa de atualização em medicina intensiva. AMIB. Artmed, Porto Alegre, 2011. p.371-418.

14. Mairuhu AT, Wagenaar J, Brandjes DP, van Gorp, EC. Dengue: an arthropod-borne disease of global importance. Eur J Clin Microbiol Infect Dis 6: 425-433, 2004.

15. Ministério da Saúde. 2015. Situação epidemiológica dados. Disponível em: http://portalsaude. saude.gov.br/index.php/situacao-epidemiologica-dados-dengue. Acesso em 08/05/2015.

16. Monteiro ESC, Coelho ME, Cunha IS, Cavalcante MAS, Carvalho FAE. Epidemiological and vector-related indicators of dengue fever in Teresina city, Piaui State, Brazil, from 2002 to 2006. Epidemiol Serv Saúde 4: 365-374, 2009.

17. Murray NEA, Quam MB, Wilder-Smith, A. Epidemiology of dengue: past, present and future prospects. Clin Epidemiol 5: 299-309, 2013.

18. Neto VSG, Rebêlo JMM. Aspectos epidemiológicos do dengue no Município de São Luís, Maranhão, Brasil, 1997-2002. Cad Saúde Pública 5: 1424-1431, 2004.

19. Neto VSG, Monteiro SG, Gonçalves AG, Rebêlo JMM. Conhecimentos e atitudes da população sobre dengue no Município de São Luís, Maranhão, Brasil, 2004. Cad Saúde Pública 22: 2191-2200, 2006.

20. O Estado do Maranhão. O Estado do Maranhão -São Luís, 16 de março de 2011 - quartafeira. Disponível em: http://imirante.com/oestadoma/online/16032011/pdf/P05.PDF. Acesso em: 12/03/2016.

21. Ribeiro AF, Marques GRAM, Voltolini JC, Condino MLF. Associação entre incidência de dengue e variáveis climáticas. Rev Saúde Pública 4: 671-676, 2006.

22. Silva FDV, Soares DL, Jesus SMC, Caldas AJM, Aquino DMC, Monteiro CA. Manifestações clínicas da febre hemorrágica do dengue associada aos principais sorotipos virais. Rev Pesq Saúde 11: 51-54, 2010.

23. Silva SS, Rangel MES. 2014. Análise quantitativa dos casos de dengue no distrito sanitário do Bequimão e da COHAB / São Luis - MA (2011-2012). $2^{\circ}$ Simpósio Brasileiro de Saúde \& Ambiente. Belo Horizonte/MG. Disponível em: http://www.sibsa.com.br/resources/ anais/4/1404184567_ARQUIVO_2SimposioBrasileirodeSaude.pdf. Acesso em 13/03/2016.

24. Secretaria Estadual de Saúde do Maranhão. SES treina enfermeiros e coordenadores municipais para ações de combate a dengue, 2014. Disponível em: http://www.saude.ma.gov.br/. Acesso em 15/05/2015.

25. Tauil PL. Urbanização e ecologia do dengue. Cad Saúde Pública S17: 99-102, 2001.

26. Vasconcelos PFC, Lima JWO, Raposo ML, Rodrigues SG, Rosa JST, Amorim SMC. Inquérito soro-epidemiológico na Ilha de São Luís durante epidemia de dengue no Maranhão. Rev Soc Bras Med Trop 32: 171-179, 1999.

27. Viana DV, Ignotti E. The ocurrence of dengue and weather changes in Brazil: A systematic review. Rev Bras Epidemiol 16: 240-256, 2013. 